\title{
Anatomy of the superior hypogastric plexus and its application in nerve-sparing paraaortic lymphadenectomy
}

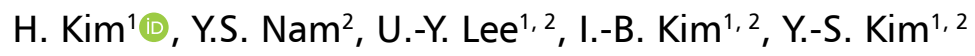 \\ ${ }^{1}$ Department of Anatomy, College of Medicine, Catholic University of Korea, Seoul, Korea \\ ${ }^{2}$ Catholic Institute for Applied Anatomy, College of Medicine, Catholic University of Korea, Seoul, Korea
}

[Received: 7 February 2020; Accepted: 16 February 2020]

\begin{abstract}
Background: The purpose of this study was to clarify the anatomy of the superior hypogastric plexus, which would contribute to advancement of nerve-sparing paraaortic lymphadenectomy.

Materials and methods: Eighteen cadavers were dissected and morphometrically analysed based on photographic images. Anatomical landmarks such as aortic bifurcation, transitional points of abdominal aorta to bilateral common iliac arteries, and cross point of the right ureter and pelvic brim, and cross point of sigmoid mesentery and pelvic brim were selected as reference points.

Results: The left lowest lumbar splanchnic nerve was located more laterally to transitional point of abdominal aorta to in 11/18 specimens, whereas the right lowest lumbar splanchnic nerve passed onto the right transitional point in only one specimen. The lowest lumbar splanchnic nerves or the superior hypogastric plexus covered the aortic bifurcation in 11/18 specimens. The superior hypogastric plexus was separate from the cross point of right ureter and pelvic brim as well as cross point of sigmoid mesentery and pelvic brim.

Conclusions: The superior hypogastric plexus is at risk of injury during paraaortic lymphadenectomy because of its topography. Preservation of the superior hypogastric plexus regarding its anatomic basis during paraaortic lymphadenectomy is required. (Folia Morphol 2021; 80, 1: 70-75)
\end{abstract}

Key words: lowest lumbar splanchnic nerves, nerve-sparing surgery, superior hypogastric plexus

\section{INTRODUCTION}

The superior hypogastric plexus (SHP) is a preaortic nervous plexus formed by two lateral roots and one median root [17] around the level of aortic bifurcation [18]. The lateral roots originate mainly from the lowest lumbar splanchnic nerves (LSNs) [2] and contain a sympathetic component [17]. The median root, or a continuation of the abdominal aortic plexus from the inferior mesenteric plexus [15, 17], contains both sympathetic and parasympathetic components [12]. The SHP is divided into paired hypogastric nerves at the same level or below the sacral promontory [15, 17, 18, 21].

Paraaortic lymphadenectomy is a surgical procedure normally performed concurrently with hysterectomy for diagnostic and therapeutic purposes [19]. Postoperative complications such as urinary, defecato$r y$, and sexual disorders following paraaortic lymphadenectomy have been reported $[3,4,19]$. These postoperative complications are believed to be caused by autonomic denervation during the surgery [19]. Thus, nerve-sparing methods have been established, most of 


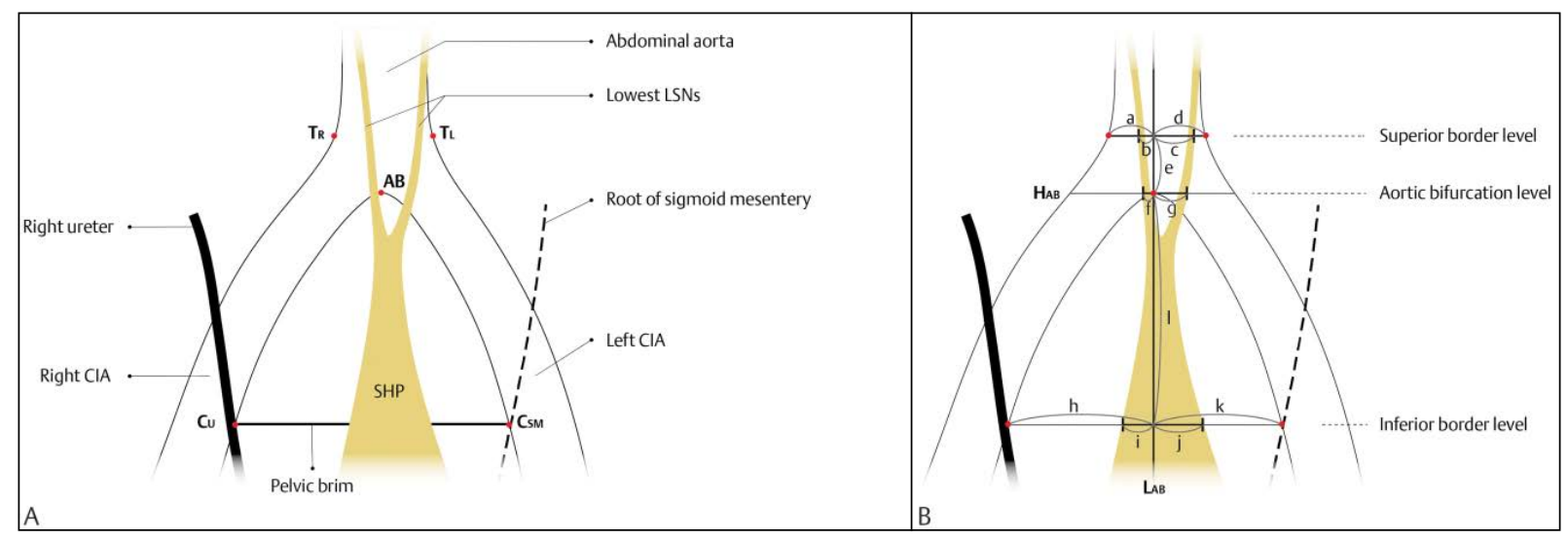

Figure 1. A. Anatomical landmarks (indicated by red dots) from the abdominal aorta and the common iliac arteries used in this study. B. Definition of 12 measurements to represent relative location of superior hypogastric plexus (SHP) and lowest lumbar splanchnic nerves (LSNs) to anatomic landmarks; $\mathrm{CIA}$ - common iliac artery; $\mathrm{AB}$ - aortic bifurcation of the abdominal aorta; $T_{R}$ - transitional point of the abdominal aorta to the right CIA; $T_{L}$ - transitional point of the abdominal aorta to the left $\mathrm{CIA} ; \mathrm{C}_{U}$ - cross point of the right ureter and the pelvic brim, $\mathrm{C}_{S M}$ - cross point of the sigmoid mesentery and the pelvic brim; $\mathrm{H}_{A B}-$ a horizontal line passing the aortic bifurcation; $\mathrm{L}_{A B}-$ a longitudinal line passing the aortic bifurcation; a- $d$ were measured at the superior border level; $f$ and $g$ were measured at the aortic bifurcation level; $\mathrm{h}-\mathrm{k}$ were measured at the inferior border level.

which focus on preservation of pelvic autonomic nerves including hypogastric nerves, pelvic splanchnic nerves, and the inferior hypogastric plexus and its visceral branches $[10,13]$. However, autonomic denervation-related complications following non-gynecologic surgery that did not involve the true pelvis but the paraaortic area have been reported $[8,9]$. These imply that autonomic denervation-related complications arising after paraaortic lymphadenectomy accompanied by hysterectomy could also be caused by injury to more superiorly located nervous structure, or SHP.

Most previous morphometric studies describing the location of the SHP adopted the midpoint of the sacral promontory [18] or the midsagittal plane [17, 20] as reference points. However, because the sacral promontory is covered by the retroperitoneum, the common iliac vessels and fatty tissue between them, and the anterior longitudinal ligament, it is not clearly observable before being fully dissected. If surgical circumstances, particularly laparoscopic, limit the opportunity for manual palpation, determining the accurate midpoint of the sacral promontory can be a significant challenge. Therefore, easily identifiable anatomical landmarks during the surgery would enhance morphometric analysis of the SHP and clinical applications to laparoscopic surgery.

The purpose of this study was to clarify the anatomy of the SHP by providing relative position of the SHP with morphometric data relevant to anatomical landmarks, which would contribute to advancement of nerve-sparing techniques surgery.

\section{MATERIALS AND METHODS}

The institutional review board of the Catholic University of Korea ruled that a cadaveric study is beyond its review authority. All cadavers used in this study were donated to the Catholic Institute for Applied and Clinical Anatomy under informed consent. Exclusion criteria included any gross evidences of retroperitoneal lesions such as aortoiliac lymph nodes metastasis and vascular malformations, and abdominopelvic surgeries, which could affect quantitative measurements. Eighteen specimens were morphometrically analyzed. Ages at death of specimens were $83.2 \pm 6.0$ years (range 69.5-91.9). Height and weight of the specimens were $153.7 \pm 6.6 \mathrm{~cm}$ (range 142.0-168.0) and $43.4 \pm 11.0 \mathrm{~kg}$ (range 26.0-64.0), respectively. Calculated body mass index was $18.3 \pm 3.9 \mathrm{~kg} / \mathrm{m}^{2}$ (range 11.3-25.0).

The method of Paraskevas et al. [17], which involves dissection of the trigone formed by the common iliac arteries and the sacral promontory, was modified. A more enlarged trapezoid-shape incision on the retroperitoneum was required to clarify the positional relationship of the SHP and anatomical landmarks adopted in this study. Five points from the abdominal aorta and the common iliac arteries were palpated and pinned (Fig. 1A). The retroperitoneum overlying the trapezoid formed by the four points, except the aortic bifurcation point, was removed with minimal structural changes. The inferior mesenteric plexus accompanying the inferior mesenteric artery was pushed away to the left. The fatty tissue overlying 
Table 1. Distances from the aortic bifurcation point to the apices of the trapezoid and the preaortic plexus formed by the lowest lumbar splanchnic nerves and the superior hypogastric plexus. Data were displayed with millimeter $(\mathrm{mm})$ scale

\begin{tabular}{|c|c|c|c|c|c|}
\hline Symbol & Description & Coordinate & Average & SD & Range \\
\hline \multicolumn{6}{|c|}{ At the superior border of the trapezoid level } \\
\hline a & To right upper apex & $x$ & -10.2 & 4.9 & $-21.2 \sim-3.1$ \\
\hline$b$ & To outer border of right lowest LSN & $x$ & -3.3 & 5.2 & $-12.8 \sim 7.5$ \\
\hline c & To outer border of left lowest LSN & $x$ & 8.8 & 4.4 & $-2.2 \sim 14.8$ \\
\hline$d$ & To left upper apex & $x$ & 10.5 & 3.8 & $0.4 \sim 14.8$ \\
\hline e & To the superior border of the trapezoid & y & 12.9 & 3.7 & $4.2 \sim 18.4$ \\
\hline \multicolumn{6}{|c|}{ At the aortic bifurcation level } \\
\hline$f$ & To outer border of the right lowest LSN & $x$ & -1.3 & 4.0 & $-9.6 \sim 5.4$ \\
\hline g & To outer border of the left lowest LSN & $x$ & 7.6 & 3.7 & $-0.8 \sim 13.5$ \\
\hline \multicolumn{6}{|c|}{ At the inferior border of the trapezoid level } \\
\hline $\mathrm{h}$ & To the right lower apex & $x$ & -34.0 & 7.8 & $-45.6 \sim-16.1$ \\
\hline $\mathrm{i}$ & To right border of the SHP & $x$ & -6.8 & 7.3 & $-21.1 \sim 5.7$ \\
\hline j & To left border of the SHP & $x$ & 12.3 & 6.9 & $-3.5 \sim 21.0$ \\
\hline k & To the left lower apex & $\mathrm{x}$ & 25.5 & 8.2 & $11.7 \sim 46.8$ \\
\hline I & To the inferior border of the trapezoid & y & -48.5 & 13.8 & $-66.3 \sim-15.3$ \\
\hline
\end{tabular}

SD — standard deviation; LSN — lumbar splanchnic nerve; SHP — superior hypogastric plexus

the SHP was removed and the bilateral borders of the SHP were dissected precisely under an OPMI pico surgical microscope with $2.5 \times$ magnifying lens (Carl Zeiss, Oberkochen, Germany). The bilateral lowest LSNs were followed to upper limit of the trapezoid. The origin of the SHP was determined as the point at which the bilateral lowest LSNs converged. The dissected SHP was documented by a digital single-lens reflex camera (Canon EOS 650D, Tokyo, Japan) with a two-centimeter scale bar for morphometric analysis in the next step.

Adobe Photoshop CC 2018 (Adobe systems incorporated, California, US) was used to measure distances. A horizontal $\left(\mathrm{H}_{\mathrm{AB}}\right)$ and a longitudinal $\left(\mathrm{L}_{\mathrm{AB}}\right)$ line passing the aortic bifurcation point were drawn. The aortic bifurcation was regarded as the datum point of the quadrant formed by the horizontal and the longitudinal line. A horizontal line connecting the $T_{R}$ and $T_{L}$ was drawn for the superior border of the trapezoid. Another horizontal line connecting the $C_{U}$ and $C_{S M}$ was drawn for the inferior border of the trapezoid.

Inside the trapezoid, 10 points at three different levels were marked (Fig. 1B). When the lowest LSN was located more laterally to the apex on the superior border, it was not marked. At each of the three levels, horizontal distances from the $\mathrm{L}_{\mathrm{AB}}$ to each marked point were measured (Fig. 1B) and described as $\mathrm{x}$-coordinates. Marked points at right side of the
$\mathrm{L}_{A B}$ were given negative values. Likewise, longitudinal distances from the $H_{A B}$ to each level were measured and described as $y$-coordinates. Marked points below the $H_{A B}$ were given negative values. Lengths of the superior and the inferior borders were calculated, as were the horizontal distances from each apex of the trapezoid to the closer border of the lowest LSNs or the SHP.

\section{RESULTS}

Measurement values representing positional relationships of the lowest LSNs and the SHP to the aortic bifurcation are described as coordinate values in the Table 1. Horizontal distances from the four apices of the trapezoid to the lowest LSNs or the SHP are described in Table 2.

At the superior border level, the left lowest LSNs located more laterally to the left upper apex $\left(T_{L}\right)$ were observed in $11 / 18$ specimens. In the other seven, the left lowest LSN was located medially to the $T_{L}$. In one specimen, the right lowest LSN passed onto the $T_{R}$ at this level. The maximal distance from the $L_{A B}$ to the outer border of the right lowest LSN was measured as $12.8 \mathrm{~mm}$, but the maximal distance to the outer border of the left lowest LSN could not be measured because of the more lateral location of the left lowest $L S N$ to the $T_{L^{-}}$. The maximal distance from the $L_{A B}$ to the left upper apex was $14.8 \mathrm{~mm}$. 
Table 2. Lengths from the four apices of the trapezoid to the preaortic plexus. Data were displayed with millimeter (mm) scale

\begin{tabular}{|c|c|c|c|}
\hline Description & Average & SD & Range \\
\hline \multicolumn{4}{|l|}{ At the superior border } \\
\hline Horizontal distance from the right upper apex to the closer border of the lowest LSN & 6.8 & 4.7 & $0 \sim 18.2$ \\
\hline Horizontal distance from the left upper apex to the closer border of the lowest LSN & 1.7 & 2.5 & $0 \sim 7.6$ \\
\hline \multicolumn{4}{|l|}{ At the inferior border } \\
\hline Horizontal distance from the right lower apex to the closer border of the SHP & 27.2 & 8.3 & $12.0 \sim 42.1$ \\
\hline Horizontal distance from the left lower apex to the closer border of the SHP & 13.2 & 9.3 & $2.7 \sim 34.4$ \\
\hline
\end{tabular}

SD — standard deviation; LSN — lumbar splanchnic nerve; SHP — superior hypogastric plexus
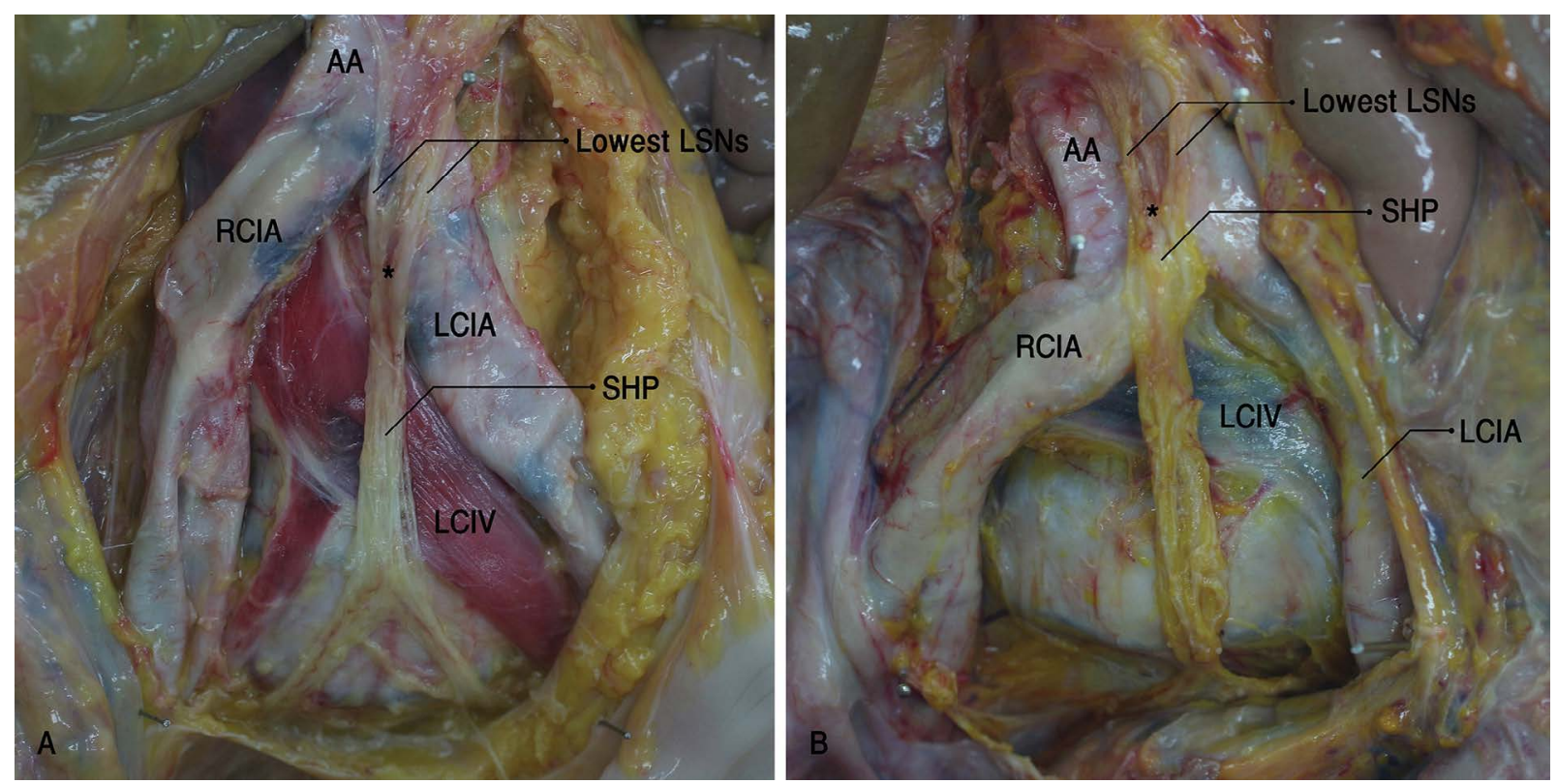

Figure 2. Ventral view of the superior hypogastric plexus (SHP); A. The SHP is formed by the bilateral lowest lumbar splanchnic nerves (LSNs) below the aortic bifurcation. The SHP is located anterior to left common iliac artery (CIA), the left common iliac vein, and the fifth lumbar vertebral body; B. The SHP is formed above the aortic bifurcation. The SHP is located anterior to the right CIA, the left common iliac vein, and the fifth lumbar vertebral body. The asterisk $\left({ }^{*}\right)$ indicates the origin of the SHP. Between the bilateral LSNs, multiple fibers from the abdominal aortic plexus were observed; AA — abdominal aorta; RCIA — right common iliac artery; LCIA — left common iliac artery; LCIV — left common iliac vein.

At the aortic bifurcation level, the preaortic plexus covered the aortic bifurcation in 11 among 18 specimens despite its slight deviation to either side. In the other cases, the entire plexus was observed to be right (1/18) or left $(6 / 18)$ of the $L_{A B}$. The maximal distances from the $L_{A B}$ to the outer border of the plexus were $9.6 \mathrm{~mm}$ right and $13.5 \mathrm{~mm}$ left.

At the inferior border level, the two lower apices $\left(C_{U}\right.$ and $\left.C_{S M}\right)$ were always separate from the SHP. The maximal distances from the $\mathrm{L}_{A B}$ to the outer border of the SHP were $21.1 \mathrm{~mm}$ right and $21.0 \mathrm{~mm}$ left. The average distance from the right ureter to the SHP was almost twice longer than the average distance from the sigmoid mesentery to the SHP.
The SHP was formed below the aortic bifurcation in 14 of 18 cases (Fig. 2A). In the other cases, the SHP was formed above the aortic bifurcation (Fig. 2B). In average, the gap between the two points at each level was narrower in the middle of the plexus than in the superior and the inferior border. The SHP occupied the widest area at the inferior border level.

\section{DISCUSSION}

The SHP was found to be at risk of injury during paraaortic lymphadenectomy because of its topography. The general appearance of the SHP was inferred through morphometric analysis in this study. The bilateral lowest LSNs converged into and formed the 
SHP below the aortic bifurcation in $14 / 18$ cases. The SHP was formed above the bifurcation, as was also reported by Ripperda et al. [18], in four specimens, but even in these cases, the origin of the SHP was located anterior to the lower portion of the abdominal aorta. The SHP, in average, occupied about one third of the space between the right ureter and the root of the sigmoid mesentery at the sacral promontory level and the width of the plexus at this level was wider than any of those at the other two levels. Briefly, the SHP was usually formed below the aortic bifurcation, located anterior to the left common iliac vessels, the fifth lumbar vertebral boy, and the sacral promontory down from above. The SHP gradually spread again like a fan from its origin before it divided into hypogastric nerves around the sacral promontory (Fig. 2A). Paraaortic lymphadenectomy involves removal of all lymph nodes around the aorta from the left renal vein superiorly to the mid-portion of the common iliac arteries inferiorly in patients with ovarian cancer or endometrial cancer [1, 11]. Depending on surgeons, the cephalad extent of paraaortic lymphadenectomy may be limited to the level of the inferior mesenteric artery for selected cases of cervical cancer [11]. In both cases of paraaortic lymphadenectomy, the area where the SHP was located is approached.

This study found that the abdominal aortic bifurcation should be avoided as an initiation point of incision during paraaortic lymphadenectomy. Because the preaortic plexus, which is composed of the bilateral LSNs and the abdominal aortic plexus anterior to the aortic bifurcation, was located between approximately $1 \mathrm{~cm}$ to the right and $1.4 \mathrm{~cm}$ left to the bifurcation in our specimens, an incision at this point [7] would damage the plexus, increasing the possibility of postoperative morbidities. For similar reasons, incisions along the bilateral borders of the abdominal aorta, particularly around the transitional points of the abdominal aorta to the common iliac arteries, are not recommended. On the left side, the lowest LSN ran down from more lateral side to the abdominal aorta and passed anterior to the left common iliac artery in usual $(11 / 18)$ cases. On the right side, one case of the lowest LSN was observed to pass onto the right transitional points. Incisions placed close to the sidewalls of the lower abdominal aorta could damage the lowest LSNs. Incisions across the right common iliac artery are worth attempting, whereas left-sided incisions could cause a problem running into the sigmoid mesentery. The SHP was about $2.7 \mathrm{~cm}$ from the cross point of the right ureter and the pelvic brim (Table 2). In van Shaik et al. [21], the SHP was reported, at the level of the sacral promontory, $2 \mathrm{~cm}$ medial and parallel to the course of the ureters, but the sample size $(n=2)$ was small. Given these observations, an initial incision placed on the retroperitoneum covering the mid-portion of the right common iliac artery would be favorable, and carrying out a longitudinal incision along the right sidewall of the inferior vena cava to the cephalad direction would be a considerable choice to avoid damage to the SHP.

This study provides positional relationships of the SHP to the abdominal aorta and the common iliac arteries. Anatomical landmarks such as the aortic bifurcation, the transitional points of the abdominal aorta to the bilateral common iliac arteries, the cross point of the right ureter and the pelvic brim, and the cross point of the sigmoid mesentery and the pelvic brim were selected for morphometric analysis. The aortic bifurcation and the right ureter were easily identifiable by inspection or palpation under the retroperitoneum even during surgery and have been used successfully as anatomical landmarks in previous radiologic $[5,16]$ and dissection studies $[18,21]$. The root of the sigmoid mesentery was selected because the left ureter was covered by the root of the sigmoid mesentery [6].

Morphometric data regarding the location and general appearance of the SHP would help clinicians identify and preserve the SHP behind the retroperitoneum, but more caution is required before direct and generalised application of this knowledge to the operation. Our results are from the specimens that were older and had lower body mass indices than the average population. This is a limitation of a cadaveric study, which completely depends on postmortem donation of materials. Radiologic studies using high-resolution magnetic resonance imaging of live human, such as magnetic resonance neurography and combined 3D reconstruction technology [14] might address this limitation.

\section{CONCLUSIONS}

This study provides clinicians with schematic information of the SHP applicable to nerve-sparing paraaortic lymphadenectomies by suggesting morphometric data with relevance to anatomical landmarks. The SHP was proven to be at risk of injury during paraaortic lymphadenectomy because of its topography. A right-sided incision from the mid-por- 
tion of the right common iliac artery to the cephalad direction along the right sidewall of the inferior vena cava is recommended to preserve the SHP. Identification and preservation of the SHP regarding its anatomic basis before lymph node dissection is required.

\section{Acknowledgements}

The authors would like to thank Eunah Hong for drawing diagrams, Jeong-Won Oh for gynecological advice, and the donors and their families without whom this research would not be possible. This research was supported by the Basic Science Research Program through the National Research Foundation of Korea (NRF) funded by the Ministry of Education (2018R1D1A1B07048476).

\section{REFERENCES}

1. Bae J, Choi JS, Lee WM, et al. Feasibility and efficacy of laparoscopic restaging surgery for women with unexpected ovarian malignancy. Eur J Obstet Gynecol Reprod Biol. 2015; 193: 46-50, doi: 10.1016/j.ejogrb.2015.06.027, indexed in Pubmed: 26232726.

2. Beveridge TS, Fournier DE, Groh AMR, et al. The anatomy of the infrarenal lumbar splanchnic nerves in human cadavers: implications for retroperitoneal nerve-sparing surgery. J Anat. 2018; 232(1): 124-133, doi: 10.1111/ joa.12721, indexed in Pubmed: 29159805.

3. Bogani G, Cromi A, Uccella $S$, et al. Nerve-sparing versus conventional laparoscopic radical hysterectomy: a minimum 12 months' follow-up study. Int J Gynecol Cancer. 2014; 24(4): 787-793, doi: 10.1097/ IGC. 0000000000000110 , indexed in Pubmed: 24552894.

4. Chen $Y, X u H$, Li $Y$, et al. The outcome of laparoscopic radical hysterectomy and lymphadenectomy for cervical cancer: a prospective analysis of 295 patients. Ann Surg Oncol. 2008; 15(10): 2847-2855, doi: 10.1245/s10434008-0063-3, indexed in Pubmed: 18649105.

5. Chithriki M, Jaibaji M, Steele RD. The anatomical relationship of the aortic bifurcation to the lumbar vertebrae: a MRI study. Surg Radiol Anat. 2002; 24(5): 308-312, doi: 10.1007/s00276-002-0036-3, indexed in Pubmed: 12497222.

6. Coffin A, Boulay-Coletta I, Sebbag-Sfez D, et al. Radioanatomy of the retroperitoneal space. Diagn Interv Imaging. 2015; 96(2): 171-186, doi: 10.1016/j.diii.2014.06.015, indexed in Pubmed: 25547251.

7. Dottino PR, Tobias DH, Beddoe A, et al. Laparoscopic lymphadenectomy for gynecologic malignancies. Gynecol Oncol. 1999; 73(3): 383-388, doi: 10.1006/gyno.1999.5376, indexed in Pubmed: 10366464.

8. Jimenez JC, Smith MM, Wilson SE. Sexual dysfunction in men after open or endovascular repair of abdominal aortic aneurysms. Vascular. 2004; 12(3): 186-191, doi: 10.1258/ rsmvasc.12.3.186, indexed in Pubmed: 15586527.

9. Johnson R, McGuire E. Urogenital complications of anterior approaches to the lumbar spine. Clin Orthop Relat Res. 1981; \&NA;(154): 114-118, doi: 10.1097/00003086$198101000-00017$.
10. Kanao H, Fujiwara K, Ebisawa K, et al. Various types of total laparoscopic nerve-sparing radical hysterectomies and their effects on bladder function. J Gynecol Oncol. 2014; 25(3): 198-205, doi: 10.3802/jgo.2014.25.3.198, indexed in Pubmed: 25045432.

11. Kavallaris A, Kalogiannidis I, Chalvatzas N, et al. Standardized technique of laparoscopic pelvic and para-aortic lymphadenectomy in gynecologic cancer optimizes the perioperative outcomes. Arch Gynecol Obstet. 2011; 283(6): 1373-1380, doi: 10.1007/s00404-010-1580-4, indexed in Pubmed: 20607263.

12. Kraima AC, van Schaik J, Susan S, et al. New insights in the neuroanatomy of the human adult superior hypogastric plexus and hypogastric nerves. Auton Neurosci. 2015; 189: 60-67, doi: 10.1016/j.autneu.2015.02.001, indexed in Pubmed: 25704391.

13. Kyo S, Kato T, Nakayama K. Current concepts and practical techniques of nerve-sparing laparoscopic radical hysterectomy. Eur J Obstet Gynecol Reprod Biol. 2016; 207: 80-88, doi: 10.1016/j.ejogrb.2016.10.033, indexed in Pubmed: 27825032.

14. Li P, Liu P, Chen C, et al. The 3D reconstructions of female pelvic autonomic nerves and their related organs based on MRI: a first step towards neuronavigation during nerve-sparing radical hysterectomy. Eur Radiol. 2018; 28(11): 4561-4569, doi: 10.1007/s00330-018-5453-8, indexed in Pubmed: 29728818.

15. Lu S, Xu Yq, Chang S, et al. Clinical anatomy study of autonomic nerve with respective to the anterior approach lumbar surgery. Surg Radiol Anat. 2009; 31(6): 425-430, doi: 10.1007/s00276-009-0461-7, indexed in Pubmed: 19190849.

16. McCullough M, Valceus J, Downes $K$, et al. The ureter as a landmark for robotic sacrocolpopexy. Female Pelvic Med Reconstr Surg. 2012; 18(3): 162-164, doi: 10.1097/ SPV.0b013e3182544e17, indexed in Pubmed: 22543768.

17. Paraskevas G, Tsitsopoulos P, Papaziogas B, et al. Variability in superior hypogastric plexus morphology and its clinical applications: a cadaveric study. Surg Radiol Anat. 2008; 30(6): 481-488, doi: 10.1007/s00276-008-0352-3, indexed in Pubmed: 18465081.

18. Ripperda CM, Jackson LA, Phelan JN, et al. Anatomic relationships of the pelvic autonomic nervous system in female cadavers: clinical applications to pelvic surgery. Am J Obstet Gynecol. 2017; 216(4): 388.e1-388.e7, doi: 10.1016/j. ajog.2016.12.002, indexed in Pubmed: 27956200.

19. Shi $R$, Wei W, Jiang P. Laparoscopic nerve-sparing radical hysterectomy for cervical carcinoma: emphasis on nerve content in removed cardinal ligaments. Int J Gynecol Cancer. 2016; 26(1): 192-198, doi: 10.1097/ IGC. 0000000000000577 , indexed in Pubmed: 26569055.

20. Shiozawa T, Huebner M, Hirt B, et al. Nerve-preserving sacrocolpopexy: anatomical study and surgical approach. Eur J Obstet Gynecol Reprod Biol. 2010; 152(1): 103-107, doi: 10.1016/j.ejogrb.2010.05.009, indexed in Pubmed: 20542624

21. van Schaik J, van Baalen JM, Visser MJ, et al. Nerve-preserving aortoiliac reconstruction surgery: anatomical study and surgical approach. J Vasc Surg. 2001; 33(5): 983-989, doi: 10.1067/mva.2001.113306, indexed in Pubmed: 11331838. 